\title{
Pasta Phase with Gyroid Morphology at Subnuclear Densities
}

\author{
Ken'ichiro Nakazato* \\ Department of Astronomy, Kyoto University, Kita-shirakawa Oiwake-cho, Sakyo, Kyoto \\ 606-8502, Japan \\ E-mail: nakazato@kusastro.kyoto-u.ac.jp
}

\section{Kazuhiro Oyamatsu}

Department of Human Informatics, Aichi Shukutoku University, Nagakute-Katahira 9, Nagakute, Aichi 480-1197, Japan

E-mail: oyakeasu.aasa.ac.jp

\begin{abstract}
About a quarter of a century ago, it was shown that nuclei deform from spheres to cylinders, slabs, cylindrical holes and spherical holes as the density increases to $\sim 10^{14} \mathrm{~g} / \mathrm{cm}^{3}$, the density of the uniform nuclear matter. These canonical pasta phases have been explicitly evaluated or implemented in many calculations, however other structures may exist. In this paper we discuss the gyroid morphology, a periodic bicontinuous structure that has also been observed in block copolymers. Earlier works used the liquid drop model to study the canonical pasta phases. Hence, we will study the gyroid morphology with the same model. The curvature effect is also taken into account perturbatively in our analysis. We then find that the gyroid morphology may appear near the transition point from a cylinder to a slab as well as that from a slab to a cylindrical hole. This interesting analogy between nuclear and polymer systems is not merely qualitative. The volume fraction at the phase transition is also similar for the two systems. Our results are also interesting because recent studies on the dynamics of pasta phases by quantum molecular dynamics show that intermediate phases, which are different from any of the known pasta phases, may emerge between the cylinder and slab phases as well as between the slab and cylindrical holes phases.
\end{abstract}

11th Symposium on Nuclei in the Cosmos, NIC XI

July 19-23, 2010

Heidelberg, Germany

\footnotetext{
${ }^{*}$ Speaker.
} 




Figure 1: Candidates for nuclear shapes. In this figure, the following notation is used: gyroid (G), gyroid hole $(\mathrm{GH})$, sphere $(\mathrm{SP})$, cylinder $(\mathrm{C})$, slab $(\mathrm{S})$, cylindrical hole $(\mathrm{CH})$ and spherical hole $(\mathrm{SH})$.

\section{Introduction}

Terrestrial nuclei are usually almost spherical while some deformations are observed to some extent. However, when they are compressed to subnuclear density, $\sim 10^{14} \mathrm{~g} / \mathrm{cm}^{3}$, in the core of supernovae or the crust of neutron stars, they are suggested to deform from spheres (SP) to cylinders $(\mathrm{C})$, slabs $(\mathrm{S})$, cylindrical holes $(\mathrm{CH})$ and spherical holes $(\mathrm{SH})$ before going into uniform matter [1, 2, 3]. Their morphologies are illustrated in Fig. 1. Since they look like meatballs, spaghetti, lasagna, macaroni and Swiss cheese, they are called "nuclear pasta". On the other hand, similar phase diagrams are obtained for nanostructures of diblock copolymers. The polymer system has an analogous order in the shape transitions. Interestingly, more complex structures are found experimentally for copolymers in the regions between cylinder and slab phases and between slab and cylindrical hole phases [4]. The gyroid (G) morphology, which is bicontinuous structure, is often thought to be a probable structure. In this study, we investigate the possible appearance of it in nuclear pasta. Further details of this study are seen in our latest paper [5] and work in progress [6].

\section{Setup and Analysis}

In this study, we utilize the liquid drop model. We settle the unit cell with the volume of $a^{3}$ and the nucleus occupies the volume fraction of $u$ in the cell. Hereafter, the term "nucleus" means the nuclear cluster. Nucleons reside in the nucleus and their number density are set to the saturation density, $n_{0}=0.165 \mathrm{fm}^{-3}$. Thus the average number density of nucleons is given as $n=u n_{0}$. The proton fraction is settled to $x_{p}=0.3$ bearing in mind the application to the core of supernovae. In 
our model, the neutron drip is not taken into account. Electrons distribute uniformly because of their large Fermi energy at the density of our interest and the their number is equal to that of protons from the charge neutrality condition. Thus the electron number density is $u x_{p} n_{0}$ both inside and outside the nucleus.

The configurations of the gyroid morphology is known to be approximated [8] as

$$
f(x, y, z)=\sin \left(\frac{2 \pi x}{a}\right) \cos \left(\frac{2 \pi y}{a}\right)+\sin \left(\frac{2 \pi y}{a}\right) \cos \left(\frac{2 \pi z}{a}\right)+\sin \left(\frac{2 \pi z}{a}\right) \cos \left(\frac{2 \pi x}{a}\right)= \pm k,
$$

where $(x, y, z)$ is the spatial coordinate and $k$ is a positive parameter. Since we assume that nucleons reside in the region $|f(x, y, z)|>k, k$ is related to the volume fraction of nucleus, $u$. We also consider the hole structure of gyroid (GH), for which nucleons reside in the region satisfying $|f(x, y, z)|<k$. This expression for the gyroid is originally introduced to approximate a minimal surface with $k=0$. While the extension for $k \neq 0$ is not the minimal surface, we call the surfaces (2.1) "gyroid" in this paper.

The total energy of our periodic cell, $W$, can be written as

$$
W=W_{\text {bulk }}+W_{\text {surf }}+W_{\text {curv }}+W_{\text {Coul }},
$$

where $W_{\text {bulk }}, W_{\text {surf }}, W_{\text {curv }}$ and $W_{\text {Coul }}$ are the bulk energy, the surface energy, the curvature energy and the Coulomb energy, respectively. Here, the bulk energy includes the electron kinetic energy inside and outside of nucleus. Since $W_{\text {bulk }}$ is proportional to the volume, we write it as

$$
W_{\text {bulk }}=w_{\text {bulk }}(u) a^{3},
$$

with the average energy density, $w_{\text {bulk }}(u)$. We assume that the surface energy is proportional to the area of the surface. Thus it depends on the shape of the nucleus whereas $W_{\text {bulk }}$ does not. We write the surface energy of a cell as

$$
W_{\text {surf }}=\sigma g(u, \text { shape }) a^{2},
$$

where $\sigma$ is the surface tension assumed to be $\sigma=0.73 \mathrm{MeV} \mathrm{fm}^{-2}$ [7]. $g(u$, shape $)$ is an area of the surface when $a=1$ with shape $=\mathrm{SP}, \mathrm{C}, \mathrm{S}, \mathrm{CH}, \mathrm{SH}, \mathrm{G}, \mathrm{GH}$. The Coulomb energy of a cell is written as

$$
W_{\text {Coul }}=\left(e x_{p} n_{0}\right)^{2} w_{\text {Coul }}(u, \text { shape }) a^{5},
$$

where $e$ is the elementary charge. $w_{\text {Coul }}(u$, shape $)$ is a relative Coulomb energy which depends on $u$ as well as on the nuclear shape. The total charge is proportional to $a^{3}$ because the charge density is fixed in our model. Since the Coulomb energy is proportional to the square of the charge and to the inverse of the length, $W_{\text {Coul }}$ is proportional to $a^{5}$.

A newly introduced term, $W_{\text {curv }}$, represents curvature corrections and we write it as

$$
W_{\text {curv }}=\omega h(u, \text { shape }) a,
$$

where $\omega$ is the curvature coefficient and $h(u$, shape $)$ is the integrated mean curvature defined as

$$
h(u, \text { shape })=\frac{1}{a} \int_{S(u, \text { shape })} H(x, y, z) \mathrm{d} S,
$$


where $H(x, y, z)$ is a mean curvature at the coordinate $(x, y, z)$ on the surface of nuclei, $S(u$, shape). Note that $h(u$, shape $)$ does not depend on $a$ because $H(x, y, z)$ is proportional to $1 / a$ and integrated over the surface. In the following, we investigate the $\omega$ dependence of the most stable shape as well as the density dependence.

Next, we put Eqs. (2.3)-(2.6) into Eq. (2.2) and minimize the energy density with respect to $a$ to obtain

$$
\frac{\partial}{\partial a}\left(\frac{W}{a^{3}}\right)=-\frac{\sigma g(u, \text { shape })}{a^{2}}-\frac{2 \omega h(u, \text { shape })}{a^{3}}+2\left(e_{p} n_{0}\right)^{2} w_{\text {Coul }}(u, \text { shape }) a=0 .
$$

Incidentally, this equation can be rewritten as $W_{\text {surf }}+2 W_{\text {curv }}=2 W_{\text {Coul }}$, which is called the condition for the size equilibrium. Since the curvature energy is small compared with the surface and Coulomb energies, we treat it perturbatively and eliminate $a$ from Eqs. (2.2) and (2.8) to get

$$
\begin{aligned}
\left(\frac{W}{a^{3}}\right)= & w_{\text {bulk }}(u)+\frac{3}{\sqrt[3]{4}}\left[\operatorname{ex}_{p} n_{0} \sigma\right]^{2 / 3} g(u, \text { shape })^{2 / 3} w_{\text {Coul }}(u, \text { shape })^{1 / 3} \\
& +\sqrt[3]{4}\left[\frac{e x_{p} n_{0} \omega^{3}}{\sigma^{2}}\right]^{1 / 3} \frac{w_{\text {Coul }}(u, \text { shape })^{2 / 3} h(u, \text { shape })}{g(u, \text { shape })^{2 / 3}} .
\end{aligned}
$$

For a given $u$, the nuclear shape is determined by minimizing the energy density in the above equation with respective to "shape".

\section{Results}

The curvature correction is largest for the nuclei with the SP morphology, followed in order by those with C, G, S, GH, CH and SH morphologies (See Fig. 3 of [6]). Note that the curvature of the $\mathrm{S}$ morphology is zero owing to its flat surface and the sign of the curvatures of hole nuclei is negative owing to their re-entrant surface. The difference between the average energy density for each shape and that of the most stable phase, $\Delta W / a^{3}$, for each shape and $\omega$ are shown in Fig. 2. $\omega$ is taken in the range $\leq 1 \mathrm{MeV} \mathrm{fm}^{-1}$ by reference to the calculations from the Skyrme interactions $[9,10]$. We can recognize that the transition density gets lower for each phase. For instance, without the curvature correction, the average energy density of the $\mathrm{C}$ and $\mathrm{S}$ morphologies become same at $n / n_{0}=0.35$. The $\mathrm{C}$ morphology has the curvature while the $\mathrm{S}$ morphology does not. Thus, the energy of the $\mathrm{S}$ morphology becomes lower than that of the $\mathrm{C}$ morphology at $n / n_{0}=0.35$.

We find that $\Delta W / a^{3}$ of the $G$ phase comes very close to zero at the transition point from the $\mathrm{C}$ phase to the $\mathrm{S}$ phase, while the $\mathrm{G}$ phase does not give the minimum for any $n$ and $\omega$. The same is true for $\mathrm{GH}$ phase at the transition point from the $\mathrm{S}$ phase to the $\mathrm{CH}$ phase. Nevertheless, the energy difference, $\sim 0.2 \mathrm{keV} \mathrm{fm}^{-3}$ or $\sim 3 \mathrm{keV}$ per nucleon, is much smaller than the temperatures in supernova cores, of the order of several MeV. In fact, the latest study of the quantum molecular dynamics suggests that intermediate phases which can not be categorized as any ordinary pasta phases appear in the regions between $\mathrm{C}$ and $\mathrm{S}$ phases and between $\mathrm{S}$ and $\mathrm{CH}$ phases [11]. While the density where the energy of $\mathrm{G}$ phase comes very close to those of the $\mathrm{C}$ and $\mathrm{S}$ phases is $n / n_{0} \lesssim 0.35$, the gyroid morphology in the block copolymer is often observed in a narrow range of the volume fraction near $\sim 0.35$ [12]. Since Coulomb screening cannot be neglected in the polymer system, a simple analogy hardly seems applicable. Our results, however, appear to suggest some common 

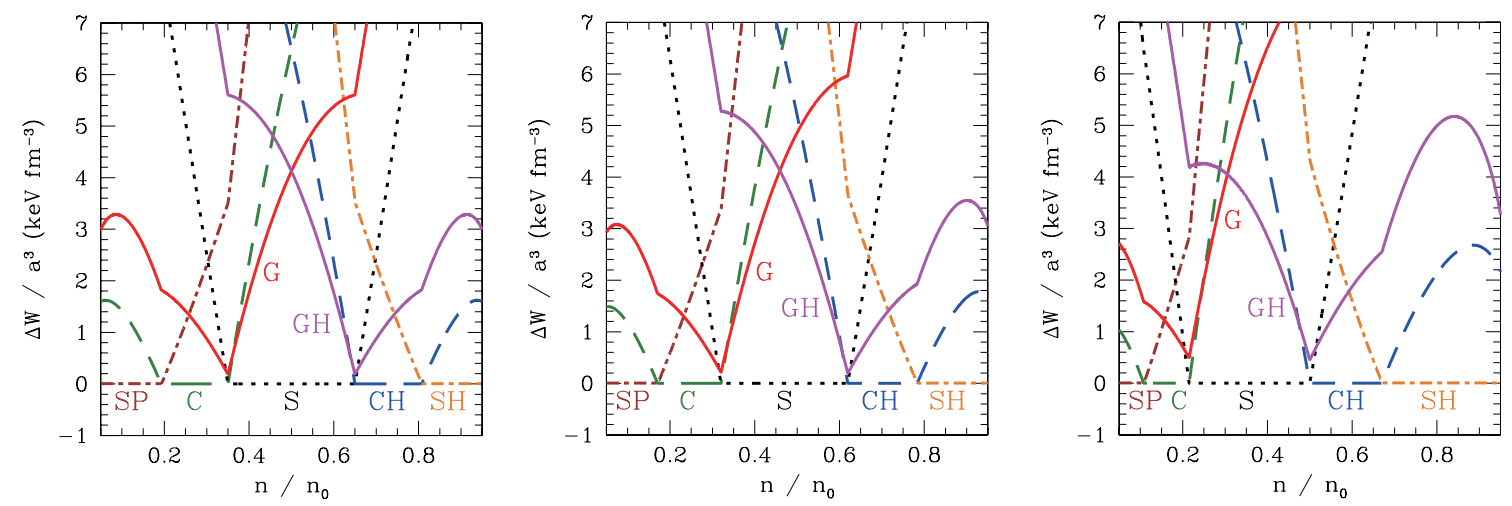

Figure 2: Differences between the average energy densities and that of the most stable phase with $\omega=$ $0 \mathrm{MeV} \mathrm{fm}^{-1}$ (left panel), $\omega=0.2 \mathrm{MeV} \mathrm{fm}^{-1}$ (central panel) and $\omega=1 \mathrm{MeV} \mathrm{fm}^{-1}$ (right panel). The notations are the same as in Fig. 1.

underlying physical principles [13]. These results are thought to imply the existence of G phase and urge to include the new member of the nuclear pasta in the detailed investigations.

\section{Acknowledgments}

We are grateful to K. Iida and S. Yamada for fruitful discussions. We also would like to thank the referee for useful comments to improve the manuscript. This work was partially supported by JSPS (K.N.).

\section{References}

[1] D.G. Ravenhall, C.J. Pethick, and J.R. Wilson, Phys. Rev. Lett. 50 (1983) 2066.

[2] M. Hashimoto, H. Seki, and M. Yamada, Prog. Theor. Phys. 71 (1984) 320.

[3] K. Oyamatsu, M. Hashimoto, and M. Yamada, Prog. Theor. Phys. 72 (1984) 373.

[4] F.S. Bates, and G.H. Fredrickson, Phys. Today 52, No. 2 (1999) 32.

[5] K. Nakazato, K. Oyamatsu, and S. Yamada, Phys. Rev. Lett. 103 (2009) 132501.

[6] K. Nakazato, K. Iida, and K. Oyamatsu, arXiv:1011.3866v2 [nucl-th].

[7] G. Watanabe, and H. Sonoda, in Soft Condensed Matter: New Research, edited by K.I. Dillon (Nova Science Publishers, New York, 2007).

[8] U. Schwarz, and G. Gompper, in Morphology of Condensed Matter: Physics and Geometry of Spatially Complex Systems, edited by K. Mecke and D. Stoyan (Springer, Berlin, 2002).

[9] K. Kolehmainen, M. Prakash, J.M. Lattimer, and J.R. Treiner, Nucl. Phys. A 439 (1985) 535.

[10] F. Douchin, P. Haensel, and J. Meyer, Nucl. Phys. A 665 (2000) 419.

[11] H. Sonoda, G. Watanabe, K. Sato, K. Yasuoka, and T. Ebisuzaki, Phys. Rev. C 77 (2008) 035806.

[12] J.H. Laurer, D.A. Hajduk, J.C. Fung, J.W. Sedat, S.D. Smith, S.M. Gruner, D.A. Agard, and R.J. Spontak, Macromolecules 30 (1997) 3938.

[13] T. Ohta, Prog. Theor. Phys. Suppl. 164 (2006) 203. 